\title{
Patellar resurfacing versus nonresurfacing in total knee arthroplasty for osteoarthritis: experience at a tertiary care institution in Pakistan
}

This article was published in the following Dove Press journal:

Open Access Surgery

13 February 2012

Number of times this article has been viewed

\author{
Akil Fazal' \\ Riaz H Lakdawala² \\ 'Clinical Fellow, NYU Hospital \\ for Joint Disease, New York, US; \\ ${ }^{2}$ Associate Professor and Chief, \\ Section of Orthopedics, Department \\ of Surgery, The Aga Khan University \\ Hospital, Karachi, Pakistan
}

Objective: To determine the effect of patellar resurfacing in patients offered total knee arthroplasty for osteoarthritis.

Design: Randomized control study.

Place and duration of study: The Aga Khan University Hospital, Karachi, Pakistan from January 3, 2005 to January 9, 2010.

Patients and methods: Patients undergoing primary total knee arthroplasty for osteoarthritis were assigned to either the patellar resurfacing or nonresurfacing arm using systematic sampling. This consisted of patients undergoing unilateral and bilateral knee arthroplasty. Preoperatively, Knee Society Knee and Function Scores were calculated. After a minimum of 3 years postoperatively Knee Society Knee and Function Scores as well as the Clinical Anterior Knee Pain Rating were calculated and analysis done to check for differences.

Results: Seventy-five patients were recruited in each arm; 135 patients had bilateral and 15 had unilateral knee arthroplasty. The mean preoperative knee score was 40.4 for the resurfacing group and 40.60 for the nonresurfacing group $(P=0.45)$. This improved postoperatively to 93.67 and 94.23 respectively, with no difference between the two groups $(P=0.67)$. The mean preoperative function score was 45.50 for resurfaced patellae and 45.83 for nonresurfaced. This improved to 89.67 and 90.50 , respectively, again with no difference $(P=0.51)$. Postoperative Clinical Anterior Knee Pain Rating was a mean of 0.1 for resurfaced and 0.13 for nonresurfaced patellas, with no difference on analysis $(P=0.06)$. However, patients who had bilateral knee arthroplasty had a slightly higher Clinical Anterior Knee Pain Rating than those who had single knee surgery $(P=0.046)$ irrespective of whether the patellar was resurfaced or not.

Conclusion: In patients undergoing primary Total Knee Arthroplasty for osteoarthritis, there is no added advantage of performing resurfacing of the patellar at 3 years of follow-up.

Keywords: patellar, patellar ligament, patellofemoral pain syndrome, arthroplasty subchondral, arthroplasy replacement knee, osteoarthritis knee, Pakistan

\section{Introduction}

The controversy about whether to resurface the patellar or to leave the native patellar unresurfaced continues to be debated by orthopedic surgeons performing total knee arthroplasties. ${ }^{1}$ When the original total knee prostheses were designed, the patellofemoral articulation was not taken into consideration as a potential source of pain, and the results were complicated by patellofemoral symptoms despite an otherwise well-performed knee arthroplasty. Subsequent designs incorporated a femoral flange for the patellofemoral articulation and provided the option for patellar resurfacing. These early implants were not designed to accommodate the native patellar in an anatomic
Correspondence: Akil Fazal

Clinical Fellow, NYU-Hospital for Joint Disease, 30I East 17th St,

New York, NY 10003, USA

Tel + I 6467305109

Fax +I 2129954154

Email akil.fazal@gmail.com
Open Access Surgery 2012:5 9-14

(C) 2012 Fazal and Lakdawala, publisher and licensee Dove Medical Press Ltd. This is an Open Access Dovepress 
fashion during the range of motion, and resurfacing of the patellar was recommended. However, complications related to patellar resurfacing became a primary concern, and have been associated with the variable revision rates often reported after total knee arthroplasty (TKA).,3

Subsequent modifications in implant design have been made to offer the surgeon the option of leaving the patellar unresurfaced. Increased awareness of component orientation and rotation has also improved the results with regard to the patellofemoral articulation after TKA. ${ }^{3}$ Numerous clinical trials have been done to help clarify the indications for patellar resurfacing. Unfortunately, there is little consensus, and surgeon preference remains the primary variable. Despite the numerous trials, there are three basic strategies: always resurface, never resurface, or selectively resurface the patellar.

\section{Methods}

This is a randomized controlled study that involved 75 patients in each arm - patellar resurfacing and nonresurfacing - who underwent primary TKA at the Aga Khan University Hospital (Karachi, Pakistan). The study period was from January 3, 2005 to January 9, 2010.

Exclusion criteria include previous patellectomy, inflammatory arthritis, patellar fracture, patellar instability, previous extensor mechanism procedures, high tibial osteotomy, severe valgus or varus instability $\left(>15^{\circ}\right)$, previous unicondylar knee replacement, and a history of septic arthritis and osteomyelitis.

All patients had Kellgren and Lawrence grade 3-4 of osteoarthritis on plain radiographs. ${ }^{4}$

Our institution has an Ethics Committee that meets every month. It is similar to an Institutional Review Board present at other institutions. It serves as a patient's advocate and is mandated with ensuring that all clinical trials are carried out within acceptable ethical standards. This trial was approved in its sitting on November 17, 2004.

All patients admitted to the orthopedic ward with osteoarthritis for TKA during the study period were identified and assigned to the relevant arm of the study. Those patients who had medical record numbers ending in even numbers were assigned to the resurfacing group. Those patients with medical record numbers ending in odd numbers were assigned to the nonresurfacing group.

A total of 150 patients were recruited; with 75 patients in each group. No power analysis was done, but this sample size is within those of other studies done on this topic..$^{5-10}$

A thorough history and physical examination was carried out by the first author (AF). All those patients fitting the exclusion criteria were withdrawn from the study. The study was explained to each patient and informed consent obtained. The preoperative Knee Society Knee and Function Scores were calculated.

Postoperative rehabilitation protocol for all patients included institution of a regimen of continuous passive motion (CPM) on the first postoperative day with a gradually progressive range of motion. Patients were also ambulated from the first postoperative day with the help of a walker. All patients were taught a quadriceps strengthening regimen in the form of straight leg raising exercises which were started in the hospital and continued for 6 weeks postoperatively. We did not use a knee brace in any of our patients.

At a minimum of 3 years postoperatively, patients were called to a free clinic and a proforma was filled out by the first author (AF) detailing post-operative Knee Society Knee and Function scores. Patients also were assessed for Clinical Anterior Knee Pain Rating as used by Waters (Figure 1). ${ }^{10}$ For bilateral knee arthroplasty patients a single data sheet was filled encompassing the patients feelings about both knees.

\begin{tabular}{|c|c|}
\hline & \multicolumn{1}{c|}{ Clinical Anterior Knee Pain Rating } \\
\hline Rating & Description \\
\hline 0 & No pain \\
I & Mild pain that does not intrude on daily activities \\
II & Moderate pain that is a nuisance; patient not \\
III & Severe pain; patient considering further surgery
\end{tabular}

Figure I Clinical anterior knee pain rating. 
The surgical approach was similar in all cases using a medial parapatellar approach. The implant used in all cases was NexGen Legacy (Zimmer, Warsaw, IN).

The Intramedullar Femoral Anterior-Posterior Sizing Guide was used to aid in setting $3^{\circ}$ of external rotation of the femoral component in relation to the nondeformed posterior condyles. The femoral and tibial components were cemented into position as per standard procedure.

\section{Patellar nonresurfacing technique}

Sharp dissection through the pre-patellar bursa to expose the anterior surface of the patellar was done. All osteophytes and synovial insertions from around the patellar were removed. Care was taken not to damage tendon insertions onto the bone.

\section{Patellar resurfacing technique}

The Universal Patellar Saw Guide was applied in line with the patellar tendon, and the patellar pushed up between its jaws. The patellar was leveled within the saw guide jaws. The amount to be resected across the top of the saw guide jaws was the same on all sides. The patellar was cut flat so that a smooth surface remained.

After centering the appropriate Patellar Drill Guide over the patellar, peg holes were then drilled into the native patellar and the artificial patellar button cemented into place.

\section{Statistics}

Data collected on the proforma was entered into SPSS (v11; IBM, New York, NY). This data was used to calculate mean and standard deviation for gender, age, height, weight, Knee Society Knee and Function Scores (pre- and postoperatively) and Clinical Anterior Knee Pain Rating (postoperative) for patellar resurfacing and nonresurfacing.

Student's $t$-test was used to detect any relationship between any two variables. A $P$-value of $<0.05$ was considered significant.

\section{Results}

\section{Demographics}

We studied a total of 150 patients, 75 in the resurfacing group and 75 in the nonresurfacing group. Analysis indicated that the groups were similar for age, duration of follow-up, gender, height, and weight (Table 1).

\section{Knee scores and function scores}

The pre-operative and post-operative knee and function score was calculated for both groups - resurfacing and nonresurfacing (Table 2). No difference was found in the two groups.
Table I Demographic results

\begin{tabular}{|c|c|c|c|}
\hline & $\begin{array}{l}\text { Resurfacing } \\
\text { group }\end{array}$ & $\begin{array}{l}\text { Nonresurfacing } \\
\text { group }\end{array}$ & $P$-value \\
\hline Age & 62.33 years & 62.07 years & 0.59 \\
\hline $\begin{array}{l}\text { Duration of } \\
\text { follow-up }\end{array}$ & 3 years 6 months & 4 years I month & 0.309 \\
\hline Gender & 52 female, 23 male & 61 female, 16 male & 0.55 \\
\hline Height & $153 \mathrm{~cm}$ & $153.8 \mathrm{~cm}$ & 0.48 \\
\hline Weight & $71.2 \mathrm{~kg}$ & $70.8 \mathrm{~kg}$ & 0.76 \\
\hline
\end{tabular}

\section{Clinical anterior knee pain rating}

Sixty-eight of the 75 patients in the resurfaced group and 70 of the 75 in the nonresurfaced group had a Clinical Anterior Knee Pain Rating postoperatively of 0, signifying no pain at all. The remaining patients had a score of 1 suggesting presence of mild pain that does not interfere with daily living.

The mean Clinical Anterior Knee Pain Rating for the resurfaced group was 0.10 and the nonresurfaced group was 0.13 , but this again was not clinically significant $(P=0.06)$. Though this is close to our significant threshold of 0.05 , as the difference was only two cases we did not treat it as significant.

\section{Unilateral versus bilateral}

Sixty-five of the 75 , or $86.6 \%$, of cases in the resurfacing group were bilateral total knee replacement whereas $13.4 \%$ were unilateral (Table 3). Seventy of the 75 , or $93.33 \%$ of cases, in the nonresurfacing group were bilateral.

Table 2 Results of clinical scores

\begin{tabular}{|c|c|c|c|}
\hline & $\begin{array}{l}\text { Resurfacing } \\
\text { group }\end{array}$ & $\begin{array}{l}\text { Nonresurfacing } \\
\text { group }\end{array}$ & $P$-value \\
\hline \multicolumn{4}{|c|}{ Preoperative Knee Society knee score results } \\
\hline Pain & 12.00 & 12.33 & 0.58 \\
\hline Stability & 22.67 & 22.83 & 0.46 \\
\hline Range of motion & 15.10 & 15.13 & 0.40 \\
\hline $\begin{array}{l}\text { Total Knee Society } \\
\text { score }\end{array}$ & 40.40 & 40.60 & 0.33 \\
\hline \multicolumn{4}{|c|}{ Postoperative Knee Society knee score results } \\
\hline Pain & 47.83 & 48.33 & 0.57 \\
\hline Range of motion & 21.53 & 21.63 & 0.26 \\
\hline Stability & 24.0 & 24.50 & 0.27 \\
\hline Total Knee Society & 93.67 & 94.23 & 0.64 \\
\hline Knee score & & & \\
\hline $\begin{array}{l}\text { Improvement in knee } \\
\text { score }\end{array}$ & $131 \%$ & $133 \%$ & \\
\hline \multicolumn{4}{|c|}{ Knee Society function score results } \\
\hline Pre-op Knee Society & 45.50 & 45.83 & 0.38 \\
\hline Function score & & & \\
\hline Post-op Knee Society & 89.67 & 90.50 & 0.51 \\
\hline
\end{tabular}


There was no difference between the groups when it came to preoperative and postoperative Knee Society Knee and Function score. However the difference between the Clinical Anterior Knee Pain Rating was significant at 0.046.

All twelve patients who reported anterior knee pain were in the bilateral group and none had unilateral knee replacements. However, as noted earlier, there was no difference between resurfaced and nonresurfaced patellas when it came to anterior knee pain.

\section{Complications}

Minor complications included deep venous thrombosis confirmed on ultrasound exams in two patients and superficial wound infection in one patient. One patient went into postoperative ileus which settled with conservative measures.

Major complications included pulmonary embolism in three patients (one of whom expired) and non-ST-elevation myocardial infarction in two patients, both of whom recovered. Out of the patients with major complications four out of five had bilateral knee surgery.

No complications related to resurfacing the patellar were encountered at the 3-year follow-up.

\section{Discussion}

Our study of 150 patients comparing patellar resurfacing versus nonresurfacing in TKA is the first study of its kind in Pakistan. As far as our demographic data was concerned, the patients were similar for age, height, weight, and gender.

The preoperative Knee Society Knee and Function Scores in the two groups were also identical, suggesting equal burden of disease in the two groups.

All patients showed tremendous improvement in Knee Society Knee and Function Scores postoperatively. It is well proved that TKA is very effective in alleviating pain and improving function in primary knee osteoarthritis.

Table 3 Unilateral and bilateral knee replacement outcomes compared

\begin{tabular}{llll}
\hline & $\begin{array}{l}\text { Unilateral } \\
(\mathbf{n}=\mathbf{I 5})\end{array}$ & $\begin{array}{l}\text { Bilateral } \\
(\mathbf{n}=\mathbf{1 3 5})\end{array}$ & P-value \\
\hline $\begin{array}{l}\text { Total preoperative Knee } \\
\text { Society knee score }\end{array}$ & 42.17 & $40.3 \mathrm{I}$ & 0.99 \\
$\begin{array}{l}\text { Total preoperative Knee } \\
\text { Society function score }\end{array}$ & 42.50 & 46.02 & 0.42 \\
$\begin{array}{l}\text { Total postoperative Knee } \\
\text { Society knee score }\end{array}$ & 93.83 & 93.96 & 0.54 \\
$\begin{array}{l}\text { Total postoperative Knee } \\
\text { Society function score }\end{array}$ & 91.66 & 89.90 & 0.29 \\
$\begin{array}{l}\text { Mean postoperative clinical } \\
\text { anterior knee pain rating }\end{array}$ & 0 & 0.13 & 0.046 \\
\hline
\end{tabular}

However, there was no difference in the improvement of the scores or the absolute postoperative knee scores in the two groups. This challenges the traditional belief that patello-femoral arthritis plays a very important part in the symptomatology of tri-compartmental osteoarthritis.

Similarly, no significant differences were found in anterior knee pain between the two groups when it came to the Clinical Anterior Knee Pain Rating. One would expect that if the patellar played a significant role in symptoms of osteoarthritis, anterior knee pain should have been significantly higher in the nonresurfaced group.

However, those patients who had bilateral TKA had a slightly higher incidence of anterior knee pain irrespective of whether the patellar was replaced or not. This may be related to them generally having a more aggressive and severe form of osteoarthritis than those with unilateral pathology.

We know of five randomized, controlled, blinded clinical trials that were performed to better answer the question of whether to resurface or to not resurface ${ }^{3,5-8}$ (Table 4). These five studies included a total of 471 knees treated with either patellar resurfacing or nonresurfacing. These randomized studies were well designed, with randomization of patients and blinding of both the examiners and the patients to the type of patellar management.

However, despite this rigorous attention to detail, the results are again variable.

Burnett et al reported a prospective, randomized, doubleblind study comparing resurfacing with nonresurfacing in 1995. ${ }^{11}$ Outcome measures included the Knee Society clinical rating, ${ }^{12}$ radiographic analysis, a 30 second stair-climbing test, and measurements of quadriceps and hamstring torque. ${ }^{13}$ The authors concluded that there was no benefit to resurfacing the patellar in knees with osteoarthritis and no serious deformity, and they did not recommend routine resurfacing of the patellar in primary TKA for osteoarthritis.

Schroeder-Boersch et $\mathrm{al}^{8}$ performed a randomized trial of 40 knees, treated with or without resurfacing, followed for a minimum of 2 years. When the patients who had a revision were excluded from the analysis of the Knee Society scores, the two groups were similar with regard to those scores for function and stair-climbing ability, although not for pain.

In 1997, Barrack et a ${ }^{14}$ reported the results of a prospective, randomized, double-blind study of 118 knees followed for a minimum of 2 years. All patients had been treated with a Miller-Galante II cruciate-retaining knee prosthesis (Zimmer, Warsaw, IN) with a femoral component featuring a nonanatomic patellofemoral groove. No difference in the overall Knee Society score or the subscore for pain or 


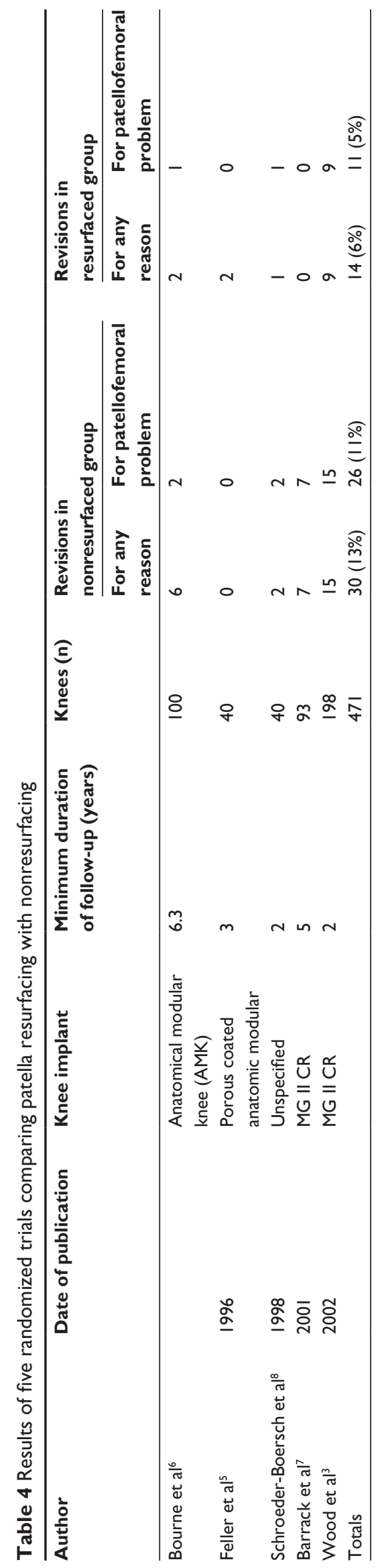

function was noted between the knees that had had patellar resurfacing and those that did not.

In 1996, Feller et $\mathrm{al}^{5}$ reported a prospective randomized trial of 40 patients without severe deformity of the patellar who underwent TKA (described as a technically uncomplicated TKA) for the treatment of osteoarthritis by a single surgeon in Australia. The authors concluded that the prevalence of anterior knee pain after TKA was not influenced by whether the patellar had been resurfaced.

In another report on 93 knees, Barrack et $\mathrm{al}^{7}$ again found no significant difference in the Knee Society pain, function, and total scores following patellar resurfacing and those following nonresurfacing. Similarly, the factors that are commonly considered for selective patellar resurfacing (obesity, degree of patellar chondromalacia, and preoperative anterior knee pain) did not predict either a lower postoperative knee score or postoperative knee pain at this later follow-up evaluation.

The largest randomized, controlled, clinical trial of which we are aware was recently performed in Australia, ${ }^{3}$ and the results differed from those of Barrack et al. The Australian study included 198 osteoarthritic knees treated with TKA with use of a Miller-Galante II implant. At a minimum of 3 years postoperatively, no difference in the Knee Society clinical rating, range of motion, or functional score was noted between the resurfaced and nonresurfaced knees. However, $31 \%$ of the nonresurfaced knees had anterior pain postoperatively compared with $16 \%$ of the resurfaced knees. The authors concluded that the results for anterior knee pain and stair descent favor the use of resurfacing and that revision rates for patellofemoral symptoms may be similar for the two techniques.

As we finish our study, it is logical for us to concur with the findings of Bennet, Barrack, and Feller. Our analysis has clearly indicated that patients with osteoarthritis who underwent TKA did equally well irrespective of whether the patellar was resurfaced or not. Though there were no added complications with resurfacing the patellar in our study, numerous other studies have reported serious complications such as patellar fracture, disruption of the extensor mechanism, osteonecrosis, aseptic loosening, instability and dislocation, "overstuffing" of the patellofemoral joint, catastrophic failure, patellar polyethylene wear, and patellar clunk syndrome. ${ }^{15}$

We recognize that the present study was of medium duration (minimum 3-year follow-up) and that accelerated failure may occur in either group with longer follow-up. The results of the present study may be specific to the type 
of prosthesis used, and different results may be reported for different designs.

Also, randomization was done using medical record numbers, which has the benefit of being easy and inexpensive. However, more accurate and sophisticated methods exist that could further reduce bias. Radiological analysis in addition to clinical data would have given further guidance on interpreting outcome.

The shortcomings of this study notwithstanding, the medium term data show no benefit in resurfacing the patellar in patients undergoing TKA for uncomplicated knee osteoarthritis.

\section{Conclusion}

\section{At 3 years follow-up}

- There is no difference in improvement of Knee Society Knee Scores or Function Scores of patients undergoing patellar resurfacing versus nonresurfacing in TKA.

- There is no difference in postoperative clinical anterior knee rating of patients undergoing patellar resurfacing versus nonresurfacing in TKA.

- Patients who underwent bilateral total knee replacement had higher incidence of anterior knee pain irrespective of whether the patellar was resurfaced or not.

- In our experience we do not think that patellar resurfacing is required in TKA procedures for osteoarthritis.

- Our indications for patellar resurfacing are inflammatory arthropathy, previous patellar fracture or dislocation, severe patellar maltracking, previous unicondylar knee arthroplasty or high tibial osteotomy, and non-anatomic tochlear groove on femoral component.

\section{Disclosure}

The authors report no conflicts of interest in this work.

\section{References}

1. Insall J, Tria AJ, Scott WN. The total condylar knee prosthesis: the first 5 years. Clin Orthop Relat Res. 1979;145:68-77.

2. Boyd AD, Ewald FC, Thomas WH, Poss R, Sledge CB. Long-term complications after total knee arthroplasty with or without of the resurfacing patella. J Bone Joint Surg Am. 1993;75(5):674-681.

3. Wood DJ, Smith AJ, Collopy D, White B, Brankov B, Bulsara MK. Patellar resurfacing in total knee arthroplasty: a prospective, randomized trial. J Bone Joint Surg Am. 2002;84-A(2):187-193.

4. Kellgren JH, Lawrence JS. Radiological assessment of osteo-arthrosis. Ann Rheum Dis. 1957;16(4):494-501.

5. Feller JA, Bartlett RJ, Lang DM. Patellar resurfacing versus retention in total knee arthroplasty. J Bone Joint Surg Br. 1996;78(2):226-228.

6. Bourne RB, Rorabeck CH, Vaz M, Kramer J, Hardie R, Robertson D. Resurfacing versus not resurfacing the patella during total knee replacement. Clin Orthop Relat Res. 1995;(321):156-161.

7. Barrack RL, Bertot AJ, Wolfe MW, Waldman DA, Milicic M, Myers L. Patellar resurfacing in total knee arthroplasty. A prospective, randomized, double-blind study with five to seven years of follow-up. J Bone Joint Surg Am. 2001;83-A(9):1376-1381.

8. Schroeder-Boersch H, Scheller G, Fischer J, Jani L. Advantages of patellar resurfacing in total knee arthroplasty. Two-year results of a prospective randomized study. Arch Orthop Trauma Surg. 1998;117(1-2):73-78.

9. Schroeder-Boersch H, Scheller G, Synnatschke M, Arnold P, Jani L. Patellar resurfacing. Results of a prospective randomized study. Orthopade. 1988;27(9):642-650. German.

10. Waters TS, Bentley G. Patella resurfacing in total knee arthroplasty. A prospective, randomized study. J Bone Joint Surg Am. 2003;85-A(2): 212-217.

11. Burnett RS, Haydon CM, Rorabek CH, Bourne RB. Patella resurfacing versus nonresurfacing in total knee arthroplasty: results of a randomized controlled clinical trial at a minimum of 10 years' followup. Clin Orthop Relat Res. 2004;428:12-25.

12. Insall JN, Dorr LD, Scott RD, Scott WN. Rationale of the Knee Society clinical rating system. Clin Orthop Relat Res. 1989;248:13-14.

13. Kramer JF. Reliability of knee extensor and flexor torques during continuous concentric-eccentric cycles. Arch Phys Med Rehabil. 1990;71(7):460-464.

14. Barrack RL, Wolfe MW, Waldman DA, Milicic M, Bertot AJ, Myers L. Resurfacing of the patella in total knee arthroplasty. A prospective, randomized, double-blind study. J Bone Joint Surg Am. 1997;79(8):1121-1131.

15. Kaufer H, Matthews LS. Spherocentric arthroplasty of the knee. Clinical experience with an average four-year follow-up. J Bone Joint Surg Am. 1981;63(4):545-559.
Open Access Surgery

\section{Publish your work in this journal}

Open Access Surgery is an international, peer-reviewed, open access journal that focuses on all aspects of surgical procedures and interventions. Patient care around the peri-operative period and patient outcomes post surgery are key topics. All grades of surgery from minor cosmetic interventions to major surgical procedures are covered. Novel techniques Submit your manuscript here: http://www.dovepress.com/open-access-surgery-journal

\section{Dovepress}

and the utilization of new instruments and materials, including implants and prostheses that optimize outcomes constitute major areas of interest. The manuscript management system is completely online and includes a very quick and fair peer-review system. Visit http://www.dovepress.com/ testimonials.php to read real quotes from published authors. 